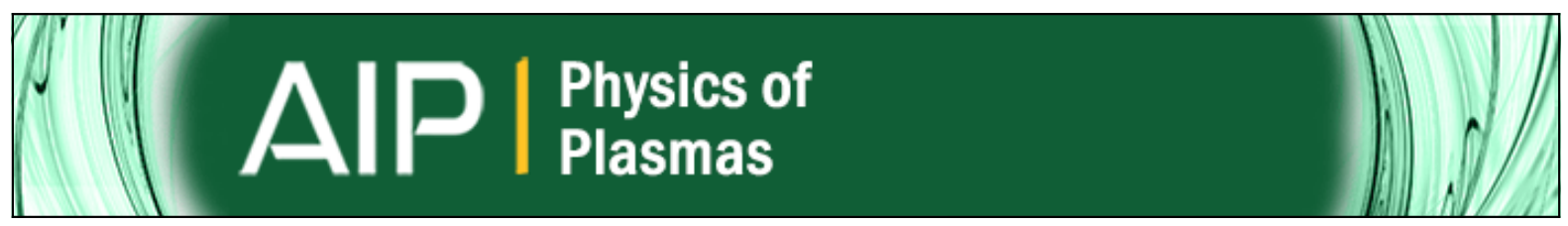

Resonances and oscillatory behavior near multi-species plasma equilibria

Manuel Núñez

Citation: Physics of Plasmas (1994-present) 21, 032117 (2014); doi: 10.1063/1.4867179

View online: http://dx.doi.org/10.1063/1.4867179

View Table of Contents: http://scitation.aip.org/content/aip/journal/pop/21/3?ver=pdfcov

Published by the AIP Publishing

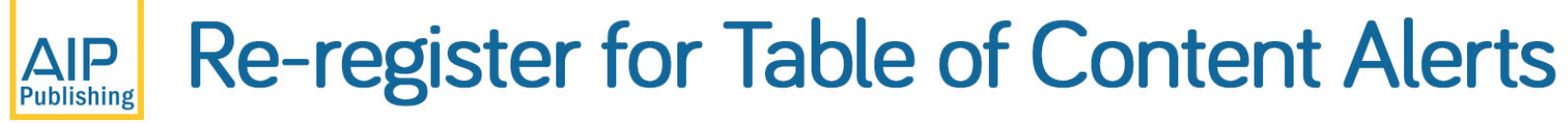

Create a profile.

Sign up today! 


\title{
Resonances and oscillatory behavior near multi-species plasma equilibria
}

\author{
Manuel Núñez ${ }^{\text {a) }}$ \\ Department of Algebra and Mathematical Analysis and IMUVA, Universidad de Valladolid, \\ 47005 Valladolid, Spain
}

(Received 16 December 2013; accepted 17 February 2014; published online 20 March 2014)

\begin{abstract}
We consider dynamic multi-species plasma equilibria whose variables depend on a single spatial coordinate and linear perturbations of these. The linearized system may be reduced to a second-order one satisfied by the respective fluid streamfunctions. For the two-species case, the electron mass is a parameter small enough for a WKB asymptotic analysis to be justified. It turns out that the points where either the ion or electron equilibrium velocity equals the ratio between the temporal and transversal frequencies of the perturbation are turning or singular points of the system, connecting exponentially increasing or decreasing solutions to oscillatory ones. The crucial role of singular points in the balance between the different contributions to the electron kinetic energy is explored. @ 2014 AIP Publishing LLC. [http://dx.doi.org/10.1063/1.4867179]
\end{abstract}

\section{INTRODUCTION}

Plasmas are often described as single fluids whose motion satisfies the Navier-Stokes equations and are governed by the Lorentz force. The electric and magnetic fields satisfy the Maxwell equations with a current density given by the flow of free charges. This is the simplest and probably the most useful model, in particular when one assumes a single neutral fluid and the natural plasma frequencies are low enough to ignore the displacement current; this assumption yields the magnetohydrodynamic approximation. However, several important phenomena such as fast magnetic reconnection ${ }^{1-4}$ cannot be adequately explained except by separation of ions and electrons. The equations governing the motion of all species of particles are conceptually simple: each of them is considered as a different fluid, satisfying the Navier-Stokes (or for inviscid fluids, the Euler) equation. All species are linked by a common electromagnetic forcing, while the electromagnetic field is generated by the flow of charged particles. Additionally, a collisional damping is present,${ }^{5}$ although many astrophysical plasmas are so rarified that can be considered effectively collisionless as well as inviscid. ${ }^{6}$ The fluid equations may be obtained by taking averages of the kinetic Vlasov equations. ${ }^{7,8}$ The full equations may be found in Ref. 9, and in a slightly simplified form in Ref. 10. Other approaches include the interpretation of plasmas as dielectric media. ${ }^{11,12} \mathrm{We}$ will first consider the existence of dynamic equilibria of a certain kind for a plasma with any number of species, but for the study of linear perturbations of those equilibria, it is convenient to take the often used model of plasmas formed by three species: ions (taken as protons), electrons, and neutral particles. In the absence of collisions, the last ones will decouple from the rest to satisfy their own unforced Euler equation, so they can be ignored. For the general collisionless system, let us denote by $m_{\alpha}, q_{\alpha}, n_{\alpha}$, and $\rho_{\alpha}$, respectively, the mass, electric charge, number density, and material density $\left(\rho_{\alpha}=m_{\alpha} n_{\alpha}\right)$ of the $\alpha$ species, $\mathbf{v}_{\alpha}$ will be its velocity, and $\mathbf{E}$ and $\mathbf{B}$ will denote,

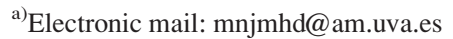

respectively, the electric and magnetic fields; finally let $P_{\alpha}$ be the kinetic pressure. Then the following equations hold:

$$
\begin{gathered}
\frac{\partial \mathbf{v}_{\alpha}}{\partial t}+\mathbf{v}_{\alpha} \cdot \nabla \mathbf{v}_{\alpha}=\frac{q_{\alpha}}{m_{\alpha}}\left(\mathbf{E}+\mathbf{v}_{\alpha} \times \mathbf{B}\right)-\frac{\nabla P_{\alpha}}{\rho_{\alpha}}, \\
\frac{\partial \mathbf{B}}{\partial t}=-\nabla \times \mathbf{E}, \\
\frac{1}{\mu_{0}} \nabla \times \mathbf{B}=\mathbf{J}+\epsilon_{0} \frac{\partial \mathbf{E}}{\partial t} .
\end{gathered}
$$

A number of additional equations must be added: a state equation relating pressure and density, and Ohm's law defining the current density $\mathbf{J}$. To simplify the analysis, we will take the fluid as incompressible, $m_{\alpha}, q_{\alpha}, n_{\alpha}$, and $\rho_{\alpha}$ as constants. Thus, we must add

$$
\begin{gathered}
\nabla \cdot \mathbf{v}_{\alpha}=0, \\
\mathbf{J}=\sum_{\alpha} n_{\alpha} q_{\alpha} \mathbf{v}_{\alpha} .
\end{gathered}
$$

Although to find equilibria the following hypothesis is unnecessary, later we will admit one of the MHD assumptions: that due to the low frequencies present, the displacement current is vanishingly small, which mathematically is equivalent to take $\epsilon_{0}=0$ in (3)

$$
\frac{1}{\mu_{0}} \nabla \times \mathbf{B}=\mathbf{J}
$$

The role of the pressure is different in compressible fluids, where it is linked to the remaining thermodynamic variables by a state equation, than in incompressible ones. In the last case, it plays an analogous role to a Lagrange multiplier, its purpose being to ensure that the remaining terms in (1) form an irrotational field (see, e.g., Ref. 13). In the compressible case, the continuity equation for each number density $n_{\alpha}$ must be added, as well as the state equation relating $P_{\alpha}$ to $n_{\alpha}$ and the temperature $T_{\alpha} . T_{\alpha}$ satisfies another evolution equation governed by the heat flux (see Ref. 9). In order to omit the pressure, it is convenient to take the curl of (1). Denote 
by $\omega_{\alpha}=\nabla \times \mathbf{v}_{\alpha}$ the vorticity of the $\alpha$-fluid. Then it is shown in Ref. 14 that (1) may be changed to

$$
\frac{\partial}{\partial t}\left(\omega_{\alpha}+\frac{q_{\alpha}}{m_{\alpha}} \mathbf{B}\right)=\nabla \times\left[\mathbf{v}_{\alpha} \times\left(\omega_{\alpha}+\frac{q_{\alpha}}{m_{\alpha}} \mathbf{B}\right)\right]
$$

(7) follows from (1)-(3) by a straightforward calculation. The converse goes as follows: provided the domain where all the spatial variables lie is simply connected, (7) may be uncurled to yield

$$
\frac{\partial \mathbf{v}_{\alpha}}{\partial t}+\mathbf{v}_{\alpha} \cdot \nabla \mathbf{v}_{\alpha}=\frac{q_{\alpha}}{m_{\alpha}}\left(\mathbf{E}+\mathbf{v}_{\alpha} \times \mathbf{B}\right)-\nabla F_{\alpha}
$$

for some potential $F_{\alpha}$. If we define now the pressure $P_{\alpha}$ as

$$
P_{\alpha}=\rho_{\alpha} F_{\alpha}-\frac{1}{2} \rho_{\alpha} v_{\alpha}^{2}
$$

we recover (1).

\section{ONE-DIMENSIONAL EQUILIBRIA AND LINEARIZED EQUATIONS}

Solutions of (2), (4)-(7) not depending on time must satisfy

$$
\begin{gathered}
\nabla \times\left[\mathbf{v}_{\alpha} \times\left(\omega_{\alpha}+\frac{q_{\alpha}}{m_{\alpha}} \mathbf{B}\right)\right]=0 \\
\nabla \times \mathbf{E}=\mathbf{0} \\
\nabla \times \mathbf{B}=\mu_{0} \sum_{\alpha} q_{\alpha} n_{\alpha} \mathbf{v}_{\alpha} .
\end{gathered}
$$

We will make the following assumptions: all the magnitudes will depend only on the variable $x \in[0, \infty)$, the velocity and the electric field will have the direction of $\hat{y}$, the magnetic field the direction of $\hat{z}$. This is the cartesian version of the geometry where the particles describe circles around the $\hat{z}$ axis, and the magnetic field is vertical. Although the equilibrium could easily be found in this axisymmetric case, the linearized equations become more complex because of the presence of the radius in the derivatives. Hence, we stick to our cartesian geometry. Since (4) holds, there exists a streamfunction $\phi_{\alpha}(x)$ such that

$$
\mathbf{v}_{\alpha}=\nabla \phi_{\alpha} \times \hat{z}=\left(0,-\phi_{\alpha}^{\prime}, 0\right)
$$

In what follows the prime will denote derivative with respect to $x$. $\mathbf{E}$ plays no role in the equilibrium equations, except to note that the electric field must be irrotational. Let $\mathbf{B}=(0,0, B)$. Then

$$
\nabla \times \mathbf{B}=\left(0,-B^{\prime}, 0\right)=-\mu_{0} \sum_{\alpha} q_{\alpha} n_{\alpha}\left(0, \phi^{\prime}{ }_{\alpha}, 0\right) .
$$

Thus

$$
\mathbf{B}=\left(\mu_{0} \sum_{\alpha} q_{\alpha} n_{\alpha} \phi_{\alpha}+C\right) \hat{z}
$$

We will take the logical assumption that velocities and fields vanish as $x \rightarrow \infty$. If they do so fast enough for them to be integrable in $[0, \infty)$, we may take the streamfunctions $\phi_{\alpha}$ satisfying also $\phi_{\alpha}(\infty)=0$. With this condition, the constant $C$ equals zero. Thus

$$
\omega_{\alpha}=\left(0,0,-\phi_{\alpha}^{\prime \prime}\right)
$$

$$
\mathbf{v}_{\alpha} \times\left(\omega_{\alpha}+\frac{q_{\alpha}}{m_{\alpha}} \mathbf{B}\right)=-\phi_{\alpha}^{\prime}\left(-\phi_{\alpha}^{\prime \prime}+\mu_{0} \frac{q_{\alpha}}{m_{\alpha}} \sum_{\beta} n_{\beta} q_{\beta} \phi_{\beta}, 0,0\right) .
$$

This is a function of the form $(f(x), 0,0)$, whose curl is zero. Hence, (10)-(12) holds and all those configurations correspond to (non-static) equilibria.

Let us consider now small perturbations of this equilibrium, $\mathbf{v}_{\alpha}+\mathbf{u}_{\alpha}, \mathbf{E}+\mathbf{e}, \mathbf{B}+\mathbf{b}, P_{\alpha}+p_{\alpha}$. The density is assumed invariant, and the perturbations take the following form: all of them depend only on $(t, x, y), \mathbf{u}_{\alpha}$ lies in the $x y$ plane, $\mathbf{e}=e \hat{y}$, $\mathbf{b}=b \hat{z}$. Let

$$
\mathbf{w}_{\alpha}=\nabla \times \mathbf{u}_{\alpha}
$$

Taking (7) instead of (1) as momentum equation, the linearized equations are

$$
\begin{aligned}
& \frac{\partial}{\partial t}\left(\mathbf{w}_{\alpha}+\frac{q_{\alpha}}{m_{\alpha}} \mathbf{b}\right)= \nabla \times\left[\mathbf{v}_{\alpha} \times\left(\mathbf{w}_{\alpha}+\frac{q_{\alpha}}{m_{\alpha}} \mathbf{b}\right)\right] \\
&+\nabla \times\left[\mathbf{u}_{\alpha} \times\left(\omega_{\alpha}+\frac{q_{\alpha}}{m_{\alpha}} \mathbf{B}\right)\right], \\
& \frac{\partial \mathbf{b}}{\partial t}=-\nabla \times \mathbf{e} \\
& \frac{1}{\mu_{0}} \nabla \times \mathbf{b}=\sum_{\alpha} n_{\alpha} q_{\alpha} \mathbf{u}_{\alpha} \\
& \nabla \cdot \mathbf{u}_{\alpha}=0 .
\end{aligned}
$$

Since (22) holds, we may take a streamfunction for $\mathbf{u}_{\alpha}$

$$
\mathbf{u}_{\alpha}=\left(\frac{\partial \psi_{\alpha}}{\partial y},-\frac{\partial \psi_{\alpha}}{\partial x}, 0\right)
$$

so that

$$
\mathbf{w}_{\alpha}=-\left(\Delta \psi_{\alpha}\right) \hat{z}
$$

where $\Delta=\nabla^{2}$ is the Laplacian operator. (21) may be written as

$$
\nabla\left(b-\mu_{0} \sum_{\alpha} n_{\alpha} q_{\alpha} \psi_{\alpha}\right)=\mathbf{0}
$$

Choosing as before $\psi_{\alpha}$ such that $\psi_{\alpha}(x=\infty)=0$ and assuming $b(x=\infty)=0$, we obtain

$$
b=\mu_{0} \sum_{\alpha} n_{\alpha} q_{\alpha} \psi_{\alpha} .
$$

After some calculation, one finds

$$
\mathbf{v}_{\alpha} \times\left(\mathbf{w}_{\alpha}+\frac{q_{\alpha}}{m_{\alpha}} \mathbf{b}\right)=\left(\Delta \psi_{\alpha}-\frac{q_{\alpha}}{m_{\alpha}} \mathbf{b}\right) \nabla \phi_{\alpha}
$$




$$
\nabla \times\left[\mathbf{v}_{\alpha} \times\left(\mathbf{w}_{\alpha}+\frac{q_{\alpha}}{m_{\alpha}} \mathbf{b}\right)\right]=-\frac{\partial}{\partial y}\left(\Delta \psi_{\alpha}-\frac{q_{\alpha}}{m_{\alpha}} \mathbf{b}\right) \phi_{\alpha}^{\prime} \hat{z},
$$

as well as

$$
\begin{aligned}
\mathbf{u}_{\alpha} \times\left(\omega_{\alpha}+\frac{q_{\alpha}}{m_{\alpha}} \mathbf{B}\right) & =\left(\phi_{\alpha}^{\prime \prime}-\frac{q_{\alpha}}{m_{\alpha}} \mathbf{B}\right) \nabla \psi_{\alpha}, \\
\nabla \times\left[\mathbf{u}_{\alpha} \times\left(\omega_{\alpha}+\frac{q_{\alpha}}{m_{\alpha}} \mathbf{B}\right)\right] & =\frac{\partial}{\partial x}\left(\phi_{\alpha}^{\prime \prime}-\frac{q_{\alpha}}{m_{\alpha}} \mathbf{B}\right) \frac{\partial \psi_{\alpha}}{\partial y} \hat{z} .
\end{aligned}
$$

Hence, (19) may be written as

$$
\begin{aligned}
\frac{\partial}{\partial t}\left(\Delta \psi_{\alpha}-\mu_{0} \frac{q_{\alpha}}{m_{\alpha}} \sum_{\beta} n_{\beta} q_{\beta} \psi_{\beta}\right) \\
=\left[\frac{\partial}{\partial y}\left(\Delta \psi_{\alpha}-\mu_{0} \frac{q_{\alpha}}{m_{\alpha}} \sum_{\beta} n_{\beta} q_{\beta} \psi_{\beta}\right)\right] \phi_{\alpha}^{\prime} \\
-\left[\frac{\partial}{\partial x}\left(\phi_{\alpha}^{\prime \prime}-\mu_{0} \frac{q_{\alpha}}{m_{\alpha}} \sum_{\beta} n_{\beta} q_{\beta} \phi_{\beta}\right)\right] \frac{\partial \psi_{\alpha}}{\partial y} .
\end{aligned}
$$

We will take now a Fourier transform in the variables $(t, y) \rightarrow(\omega, m)$. Since we will later assume that any solution evolving as $\exp (i \omega t)$ is the limit of damped solutions $\exp (-\epsilon t+i \omega t)$ as $\epsilon \rightarrow 0$, we need for the functions $\exp (-\epsilon t) \psi_{\alpha}(t), \exp (-\epsilon t) \psi_{\alpha}^{\prime}(t), \exp (-\epsilon t) \psi_{\alpha}^{\prime \prime}(t)$ to be integrable in time at least for $0 \leq \epsilon<c$ for some $c>0$. This occurs, for example, if $\psi_{\alpha}$ has parabolic support, i.e., if $\psi_{\alpha}$ vanishes outside an interval $(-R, \infty)$ for some finite $R$. Alternatively, we could take the Laplace transform in time, but this needs that $\psi_{\alpha}$ as well as $\psi_{\alpha}^{\prime}$ and $\psi_{\alpha}^{\prime \prime}$ vanish at $t=0$ if we want the final equation to be homogeneous. Since $\psi_{\alpha}$ is a perturbation, this hypothesis is justified by arguing that we wish to study the evolution of this perturbation as determined by the equilibrium quantities, not by any arbitrary initial values of it.

We will denote the Fourier transform of $\psi_{\alpha}$ by $\Psi_{\alpha}$. Since $\psi_{\alpha}$ is a real function, $\Psi_{\alpha}$ satisfies

$$
\Psi_{\alpha}(-\omega, x,-m)=\overline{\Psi_{\alpha}(\omega, x, m)} .
$$

We must recall that $\Psi^{\prime}{ }_{\alpha}$ may be singular at some point even if the original function $\mathbf{u}_{\alpha}$ remains small; the singularities of the Fourier transform depend on the decreasing rate of $\psi_{\alpha}$ and may well occur even if $\psi_{\alpha}$ is smooth. Assuming the reasonable hypothesis that $\psi_{\alpha}$ as well as $\psi_{\alpha}^{\prime}$ and $\psi_{\alpha}^{\prime \prime}$ are tempered distributions, (31) transforms into

$$
\begin{aligned}
i \omega( & \left.\Psi_{\alpha}^{\prime \prime}-m^{2} \Psi_{\alpha}-\mu_{0} \frac{q_{\alpha}}{m_{\alpha}} \sum_{\beta} n_{\beta} q_{\beta} \Psi_{\beta}\right) \\
= & -i m \Psi_{\alpha}\left(\phi_{\alpha}^{\prime \prime \prime}-\mu_{0} \frac{q_{\alpha}}{m_{\alpha}} \sum_{\beta} n_{\beta} q_{\beta} \phi_{\beta}^{\prime}\right) \\
& +i m \phi_{\alpha}^{\prime}\left(\Psi_{\alpha}^{\prime \prime}-m^{2} \Psi_{\alpha}-\mu_{0} \frac{q_{\alpha}}{m_{\alpha}} \sum_{\beta} n_{\beta} q_{\beta} \Psi_{\beta}\right) .
\end{aligned}
$$

$$
\begin{gathered}
F_{\alpha}=\phi_{\alpha}^{\prime \prime \prime}-\mu_{0} \frac{q_{\alpha}}{m_{\alpha}} \sum_{\beta} n_{\beta} q_{\beta} \phi_{\beta}^{\prime}, \\
G_{\alpha}=\phi_{\alpha}^{\prime} .
\end{gathered}
$$

Then (33) may be written as a second-order differential system on the variables $\Psi_{\alpha}$

$$
\Psi_{\alpha}^{\prime \prime}-m^{2} \Psi_{\alpha}-\mu_{0} \frac{q_{\alpha}}{m_{\alpha}} \sum_{\beta} n_{\beta} q_{\beta} \Psi_{\beta}=\frac{m F_{\alpha}}{m G_{\alpha}-\omega} \Psi_{\alpha}
$$

\section{ASYMPTOTIC ANALYSIS IN THE TWO-FLUID CASE}

In order to make some progress with (36), we consider a plasma formed by monoatomic ions, electrons, and neutral particles and denote their respective streamfunctions vanishing at infinity by $\Psi_{p}, \Psi_{e}$, and $\Psi_{n}$. Since $q_{n}=0$, the equation for $\Psi_{n}$ decouples from the rest

$$
\Psi_{n}^{\prime \prime}-m^{2} \Psi_{n}-\frac{m F_{n}}{m G_{n}-\omega} \Psi_{n}=0
$$

and will be omitted. To avoid dragging constants, we take units so that $q_{p}=1, q_{e}=-1, m_{p}=1, n_{e}=n_{p}=N$. These are simplifying hypotheses and the analysis could be done with more complex parameters; the condition that the number densities coincide models a globally neutral plasma. As for $m_{e}$, we denote it by $\epsilon^{2}$; for the case electron/proton, $\epsilon^{2} \sim 5.5 \cdot 10^{-4}$, which is small enough to justify a WKB approximation for the system (37). This now takes the form

$$
\begin{gathered}
\Psi_{p}^{\prime \prime}-m^{2} \Psi_{p}-N \Psi_{p}+N \Psi_{e}-\frac{m F_{p}}{\omega-m G_{p}} \Psi_{p}=0 \\
\Psi_{e}^{\prime \prime}-m^{2} \Psi_{e}-\frac{N}{\epsilon^{2}} \Psi_{e}+\frac{N}{\epsilon^{2}} \Psi_{p}-\frac{m F_{e}}{\omega-m G_{e}} \Psi_{e}=0 .
\end{gathered}
$$

We could obtain $\Psi_{e}$ in terms of $\Psi_{p}$ from (38) and plug it into (39), obtaining a fourth-order equation in $\Psi_{p}$. This equation, however, is far less transparent than the original system. Writing down the expressions of $F_{p}, G_{p}, F_{e}$, and $G_{e}$, (38) and (39) may be set for $m \neq 0$ as

$$
\begin{aligned}
& \Psi_{p}^{\prime \prime}-\left(m^{2}+N \frac{\phi_{e}^{\prime}-\frac{\omega}{m}}{\phi_{p}^{\prime}-\frac{\omega}{m}}+\frac{\phi_{p}^{\prime \prime \prime}}{\phi_{p}^{\prime}-\frac{\omega}{m}}\right) \Psi_{p}+N \Psi_{e}=0, \\
& \Psi_{e}^{\prime \prime}-\left(m^{2}+\frac{N}{\epsilon^{2}} \frac{\phi_{p}^{\prime}-\frac{\omega}{m}}{\phi_{e}^{\prime}-\frac{\omega}{m}}+\frac{\phi_{e}^{\prime \prime \prime}}{\phi_{e}^{\prime}-\frac{\omega}{m}}\right) \Psi_{e}+\frac{N}{\epsilon^{2}} \Psi_{p}=0 .
\end{aligned}
$$

We will look for solutions of rapid variation due to the presence of the large parameter $\lambda=\sqrt{N} / \epsilon$ by the WKB method. This scheme, named after Wentzel, Kramers, and Brillouin, goes in fact much further back in time (see, e.g., Ref. 15). We will extract the results relevant to our study from three texts: the simplest but full of interesting examples is Ref. 16 . A more complete treatment including singular points and first-order systems may be seen in Ref. 17, and the classical reference $^{18}$ devotes some chapters to the WKB method. We 
have found no reference where the connection formulas through singular points are clearly written down, so we will have to reconstruct them. As asserted, there exists a standard WKB treatment for first order systems, ${ }^{17}$ but it runs into difficulties when the eigenvalues of the matrix which multiplies the large parameter $\lambda$ are not all different, as well as in the turning and singular points. Since these are the most interesting aspects of the analysis, we simply pose a WKB form of solution for (40) and (41), which we will abbreviate to

$$
\begin{gathered}
\Psi_{p}^{\prime \prime}=f \Psi_{p}-N \Psi_{e}, \\
\Psi_{e}^{\prime \prime}=\lambda^{2} H \Psi_{e}+g \Psi_{e}-\lambda^{2} \Psi_{p} .
\end{gathered}
$$

$f, g$, and $H$ do not depend on $\lambda$. They are smooth except at the points where $\phi_{p}^{\prime}-\omega / m$ or $\phi_{e}^{\prime}-\omega / m$ equal zero. The asymptotic expansion of these solutions is assumed to have the form

$$
\begin{aligned}
& \Psi_{e}(x)=e^{\lambda S(x)}\left(A_{0}(x)+\frac{A_{1}(x)}{\lambda}+\frac{A_{2}(x)}{\lambda^{2}}+\ldots\right), \\
& \Psi_{p}(x)=e^{\lambda S(x)}\left(B_{0}(x)+\frac{B_{1}(x)}{\lambda}+\frac{B_{2}(x)}{\lambda^{2}}+\ldots\right) .
\end{aligned}
$$

Taking (44) and (45) into (42) and (43), and equating the terms in $\lambda^{2}$, we obtain the eikonal system

$$
\begin{gathered}
\left(S^{\prime 2}-H\right) A_{0}=B_{0}, \\
S^{\prime 2} B_{0}=0,
\end{gathered}
$$

which yields $B_{0}=0, S^{\prime}= \pm \sqrt{H}$. Taking now the terms of order $\lambda$

$$
\begin{gathered}
S^{\prime \prime} A_{0}+S^{\prime 2} A_{1}+2 S^{\prime} A_{0}^{\prime}=B_{1}, \\
S^{\prime 2} B_{1}+2 S^{\prime} B_{0}^{\prime}=0,
\end{gathered}
$$

so that $B_{1}=0$ and the first two terms of $\Psi_{p}$ vanish; thus $\Psi_{p}$ has order $\lambda^{-2}$ as compared with $\Psi_{e}$, although the rate of variation given by $S$ is the same. Hence, for the physical optics approximation we may ignore $\Psi_{p}$ and consider only

$$
\Psi_{e}^{\prime \prime}=\lambda^{2} H \Psi_{e}+g \Psi_{e} .
$$

Notice that even the possible singularities of $f$ in (42) do not challenge this relative order provided they are at most of order two, since these are regular singularities and the solution there remains bounded. In fact, we will consider only singularities of order one, which are the only stable ones; a small perturbation of a higher order one will turn it into a number of simple ones or will make it disappear. This relative order translates into the derivatives $\Psi_{p}^{\prime}$ and $\Psi_{e}^{\prime}$, and therefore into the ion and electron velocities. The larger electron velocity is a logical consequence of its smaller mass as compared with the ions.

Equation (50) may be studied by the classical scalar WKB method. The zeroes $x_{p}$ of $H$ represent turning points, whose behavior is well known, in particular when they are of order one, as we assume. ${ }^{16}$ The poles $x_{e}$ of $H$ represent singularities, studied, e.g., in Refs. 17 and 18, and as we will see they have particular properties highly relevant to our case. The key point is the distribution of zeroes of $\phi_{p}^{\prime}-\omega / m$ and $\phi_{e}^{\prime}-\omega / m$ in the interval $x \in[0, \infty)$. Obviously, the possibilities are endless, but the essential point of the behavior of the WKB approximation in each interval bounded by two of these zeroes plus the connection formulas across them, will be captured by a representative example. We choose the following configuration: a single simple zero $x_{p}$ of $\phi_{p}^{\prime}$ $-\omega / m$ followed by a single simple zero $x_{e}$ of $\phi_{e}^{\prime}-\omega / m$, in the form $0<x_{p}<x_{e}<\infty$. This is appropriate for the reasonable assumption that the equilibrium velocity $\phi_{p}^{\prime}$ of the ions is less than the one $\phi_{e}^{\prime}$ of the electrons, and both decrease with $x$ as $x$ grows. The case where both equilibrium velocities are identical is completely different; there $H=1$ and only the singularity of $g$ may exist. We will consider this case at the end. In our case, $H$ is positive in $\left(0, x_{p}\right)$ and $\left(x_{e}, \infty\right)$, and negative in $\left(x_{p}, x_{e}\right)$.

\section{A. Exponential solutions and connections through the turning point}

The first point is that since $\Psi_{e}(\infty)=0$ and the coefficients of (50) are real, this will determine the $\Psi_{e}$ up to a multiplicative constant. In order to write down the WKB solutions in each interval, let us define

$$
\begin{aligned}
& S_{p}(x)=\int_{x_{p}}^{x} \sqrt{H(s)}, d s . \\
& S_{e}(x)=\int_{x_{e}}^{x} \sqrt{|H(s)|}, d s .
\end{aligned}
$$

Notice that $\sqrt{|H|}$ is always integrable, even in a neighborhood of $x_{e}$, and

$$
S_{p}(x)=S_{e}(x)+\int_{x_{p}}^{x_{e}} \sqrt{|H(s)|} d s=S_{e}(x)+k .
$$

Also $S_{p}(x)<0$ for $x<x_{p}, S_{e}(x)<0$ for $x<x_{e}$. A fundamental system of WKB solutions is for any small $\delta>0$

(1) $\operatorname{In}\left(0, x_{p}-\delta\right) \cup\left(x_{e}+\delta, \infty\right)$

$$
|H(x)|^{-1 / 4} e^{-\lambda\left|S_{p}(x)\right|}, \quad|H(x)|^{-1 / 4} e^{\lambda\left|S_{p}(x)\right|},
$$

(2) $\operatorname{In}\left(x_{p}+\delta, x_{e}-\delta\right)$

$$
|H(x)|^{-1 / 4} e^{-i \lambda\left|S_{p}(x)\right|}, \quad|H(x)|^{-1 / 4} e^{i \lambda\left|S_{p}(x)\right|},
$$

or

$$
|H(x)|^{-1 / 4} \cos \left(\lambda\left|S_{p}(x)\right|\right), \quad|H(x)|^{-1 / 4} \sin \left(\lambda\left|S_{p}(x)\right|\right) .
$$

We could take as well the same functions changing $S_{p}$ by $S_{e}$, since

$$
\begin{gathered}
\left|S_{p}\right|=\left|S_{e}\right|-k \quad \text { for } \quad x<x_{p}, \\
\left|S_{p}\right|=-\left|S_{e}\right|+k \quad \text { for } \quad x_{p}<x<x_{e} \\
\left|S_{p}\right|=\left|S_{e}\right|+k \quad \text { for } \quad x>x_{e}
\end{gathered}
$$


so that the functions with the exponent $\left|S_{e}\right|$ differ from the ones in (55) by multiplicative constants. Connections through the turning point $x_{p}$ are well known. The trick is to identify equation (50) near $x_{p}$ with an Airy equation and use these (or equivalently, the Bessel functions of order $1 / 3$ ) to see how the WKB solutions are continuously connected. Often, ${ }^{16}$ the connection proceeds from the exponential solutions to the right to the trigonometric ones to the left, as the realistic solution is the exponentially decreasing one as $x \rightarrow \infty$. In our case, however, we must proceed from $x<x_{p}$ to $x>x_{p}$. Since these results are abundantly explained in the literature (see, e.g., Ref. 17), we merely state them. We have

$$
\begin{aligned}
&|H|^{-1 / 4} e^{-\lambda\left|S_{p}\right|} \rightarrow 2|H|^{-1 / 4} \sin \left(\lambda\left|S_{p}\right|+\frac{\pi}{4}\right) \\
&=2|H|^{-1 / 4} \sin \left(-\lambda\left|S_{e}\right|+\lambda k+\frac{\pi}{4}\right) ; \\
&|H|^{-1 / 4} e^{\lambda\left|S_{p}\right|} \rightarrow|H|^{-1 / 4} e^{i\left(\lambda\left|S_{p}\right|+\pi / 4\right)}=|H|^{-1 / 4} e^{i\left(-\lambda\left|S_{e}\right|+\lambda k+\pi / 4\right)} .
\end{aligned}
$$

As expected, the solutions are exponentially increasing or decreasing in $\left(0, x_{p}\right)$ and oscillatory in $\left(x_{p}, x_{e}\right)$. Still the only boundary condition we have, $\Psi_{e}(\infty)=0$, has not appeared and leaves open the one-dimensional space of solutions. To determine it we must turn to the singular point at $x_{e}$.

\section{B. Connections through the singular point}

As stated, even in the texts studying the singular case, ${ }^{17,18}$ explicit connection formulas through $x_{e}$ are lacking. To obtain them, we must reconstruct the approximation procedure. Assume that the functions $\phi_{e}$ and $\phi_{p}$ are analytic. One first takes the Liouville transform

$$
\zeta=\operatorname{sgn}\left(x-x_{e}\right)\left(\int_{x_{e}}^{x} \sqrt{|H(s)|} d s\right)^{2},
$$

where sgn denotes the sign. Let

$$
\begin{gathered}
\Phi_{e}=\left(\frac{\partial \zeta}{\partial x}\right)^{1 / 2} \Psi_{e}, \\
G(x)=\left(\frac{\partial \zeta}{\partial x}\right)^{2}=4 \zeta H(x), \\
G_{1}(\zeta)=\frac{1}{G(x)^{1 / 4}} \frac{d^{2}\left(G^{1 / 4}\right)}{d \zeta^{2}}+\frac{g(x)}{G(x)} .
\end{gathered}
$$

Then $\Phi_{e}$ as a function of $\zeta$ satisfies an equation of the form

$$
\frac{d^{2} \Phi_{e}}{d \zeta^{2}}=\left(\frac{\lambda^{2}}{4 \zeta}+G_{1}(\zeta)\right) \Phi_{e}
$$

and $G_{1}$ is analytic near $\zeta=0$, so that the term $\lambda^{2} /(4 \zeta)$ is dominant for two reasons near $\zeta=0$ : $|\zeta| \ll 1$ and $\lambda^{2} \gg 1$.
Hence, we may omit $G_{1}$ from (66). The remaining equation is well known. Its solutions are for $\zeta>0$

$$
\zeta \rightarrow \sqrt{\zeta} I_{1}(\lambda \sqrt{\zeta}), \quad \zeta \rightarrow \sqrt{\zeta} K_{1}(\lambda \sqrt{\zeta})
$$

where $I_{1}$ and $K_{1}$ are Bessel functions of first order. To see how the solution behaves for $\zeta<0$, recall that the Bessel functions $I_{1}$ and $J_{1}$ are odd entire functions, satisfying

$$
J_{1}(i \zeta)=i I_{1}(\zeta)
$$

On the other hand, $K_{1}$ and $Y_{1}$ are multiform functions due to the presence of a logarithm in their power expansion. Usually, the ray $(-\infty, 0]$ is excluded from the plane in their definition (i.e., we take the principal branch of the logarithm). In these conditions, for $\zeta>0$

$$
K_{1}(i \zeta)=-\frac{\pi}{2} J_{1}(\zeta)+i \frac{\pi}{2} Y_{1}(\zeta)
$$

Thus, if we take $\sqrt{-\zeta}=i \sqrt{|\zeta|}$, the solutions in (67) evolve in the following way when going from $\zeta>0$ to $\zeta<0$

$$
\begin{aligned}
& \sqrt{\zeta} I_{1}(\lambda \sqrt{\zeta}) \rightarrow i \sqrt{|\zeta|} I_{1}(i \lambda \sqrt{|\zeta|})=-\sqrt{|\zeta|} J_{1}(\lambda \sqrt{|\zeta|}) \\
& \sqrt{\zeta} K_{1}(\lambda \sqrt{\zeta}) \rightarrow i \sqrt{|\zeta|} K_{1}(i \lambda \sqrt{|\zeta|}) \\
& \quad=i \frac{\pi}{2} \sqrt{|\zeta|}\left(-J_{1}(\lambda \sqrt{|\zeta|})+i Y_{1}(\lambda \sqrt{|\zeta|})\right) \\
& \quad=-\frac{\pi}{2} \sqrt{|\zeta|} Y_{1}(\lambda \sqrt{|\zeta|})-i \frac{\pi}{2} \sqrt{|\zeta|} J_{1}(\lambda \sqrt{|\zeta|})
\end{aligned}
$$

If instead we take $\sqrt{-\zeta}=-i \sqrt{|\zeta|}$, since all the Bessel functions are real for real argument, their value at the conjugate of $z$ is the conjugate of their value at the point $z$. Thus, without further calculation

$$
\begin{gathered}
\sqrt{\zeta} I_{1}(\lambda \sqrt{\zeta}) \rightarrow-\sqrt{|\zeta|} J_{1}(\lambda \sqrt{|\zeta|}) \\
\sqrt{\zeta} K_{1}(\lambda \sqrt{\zeta}) \rightarrow-\frac{\pi}{2} \sqrt{|\zeta|} Y_{1}(\lambda \sqrt{|\zeta|})+i \frac{\pi}{2} \sqrt{|\zeta|} J_{1}(\lambda \sqrt{|\zeta|}) .
\end{gathered}
$$

We must now decide which sign is the appropriate one. First, for $x$ near $x_{e}, x>x_{e}$

$$
H(x) \sim \frac{h_{0}}{x-x_{e}},
$$

where the value $h_{0}$ is positive, since $H$ is positive. Thus, following (62), $\zeta \sim 4 h_{0}\left(x-x_{e}\right)$, so that $\zeta>0$ means $x>x_{e}$. The point $x_{e}$ is the root of $\phi_{e}^{\prime}(x)-\omega / m=0$ for fixed $\omega$, but now we allow it to vary for times frequencies near $\omega$, and denote it by $x_{e}(\omega)$. Now, we use the previous assumption that the solution for $\omega$ must be the limit of the solutions for the same Eq. (50) with $\omega+i \epsilon, \epsilon>0, \epsilon \rightarrow 0$. If the zeroes $x_{e}(\omega+i \epsilon)$ lie in the upper half plane for small positive $\epsilon$, then $x-x_{e}(\omega+i \epsilon)$ and therefore $\zeta$ changes from $\zeta>0$ to $\zeta<0$ through the lower half plane, which means that $\sqrt{\zeta}$ changes to $-i \sqrt{|\zeta|}$. The opposite happens if $x_{e}(\omega+i \epsilon)$ lies in the lower half plane. Since 


$$
x_{e}(\omega)=\left(\phi_{e}^{\prime}\right)^{-1}\left(\frac{\omega}{m}\right)
$$

for $\operatorname{Im} \omega>0$ and $m>0$ we have

$$
\begin{aligned}
& \operatorname{Im} x_{e}(\omega)>0 \text { if } \quad\left(\phi_{e}^{\prime}\right)^{-1}\left(\frac{\omega}{m}\right)=\frac{1}{\phi_{e}^{\prime \prime}\left(x_{e}(\omega)\right)}>0, \\
& \operatorname{Im} x_{e}(\omega)<0 \text { if } \quad\left(\phi_{e}^{\prime}\right)^{-1}\left(\frac{\omega}{m}\right)=\frac{1}{\phi_{e}^{\prime \prime}\left(x_{e}(\omega)\right)}<0,
\end{aligned}
$$

whereas the opposite happens for $m<0$. Thus, for $m>0$, the solutions connect as follows: if $\phi_{e}$ is convex at $x_{e}, \phi^{\prime \prime}\left(x_{e}\right)>0$, the second solution (71) and (73) goes as

$$
\sqrt{\zeta} K_{1}(\lambda \sqrt{\zeta}) \rightarrow-\frac{\pi}{2} \sqrt{|\zeta|} Y_{1}(\lambda \sqrt{|\zeta|})+i \frac{\pi}{2} \sqrt{|\zeta|} J_{1}(\lambda \sqrt{|\zeta|})
$$

If $\phi_{e}$ is concave at $x_{e}, \phi^{\prime \prime}\left(x_{e}\right)<0$, this solution changes as

$$
\sqrt{\zeta} K_{1}(\lambda \sqrt{\zeta}) \rightarrow-\frac{\pi}{2} \sqrt{|\zeta|} Y_{1}(\lambda \sqrt{|\zeta|})-i \frac{\pi}{2} \sqrt{|\zeta|} J_{1}(\lambda \sqrt{|\zeta|})
$$

The first solutions (70) and (72) do not depend on the sign of $\operatorname{Im} \omega$. The roles are changed for $m<0$. Always an imaginary part appears for $\zeta<0$ even if the solution is real for $\zeta>0$.

To recover $\Psi_{e}$, we recall that

$$
\sqrt{\zeta(x)}=\int_{x_{e}}^{x} \sqrt{|H(s)|} d s=S_{e}(x),
$$

so that $\zeta(x)=S_{e}(x)^{2}$, and

$$
\begin{aligned}
\Psi_{e}(x) & =\left(\frac{d \zeta}{d x}\right)^{-1 / 2} \cdot \Phi_{e}(x)=\left(2 S_{e}(x) S_{e}^{\prime}(x)\right)^{-1 / 2} \Phi_{e}(x) \\
& =\frac{1}{\sqrt{2}}|H(x)|^{-1 / 4} S_{e}(x)^{-1 / 2} \Phi_{e}(x) .
\end{aligned}
$$

For each of the Bessel functions of order one $F$, we will denote by $\Phi_{e F}$ the corresponding solution of (66), i.e.,

$$
\Phi_{e F}=\sqrt{\zeta(x)} F(\lambda \sqrt{\zeta(x)})=S_{e}(x) F\left(\lambda S_{e}(x)\right),
$$

and $\Psi_{e F}$ the associated solution of (50) given by (81)

$$
\Psi_{e F}(x)=\frac{1}{\sqrt{2}}|H(x)|^{-1 / 4} S_{e}(x)^{1 / 2} F\left(\lambda S_{e}(x)\right) .
$$

It is known (see, e.g., Ref. 19) that as $x \rightarrow \infty$

$$
\begin{gathered}
I_{1}(x) \sim \frac{1}{\sqrt{2 \pi x}} e^{x}\left(1+O\left(\frac{1}{x}\right)\right), \\
K_{1}(x) \sim \frac{\pi}{\sqrt{2 x}} e^{-x}\left(1+O\left(\frac{1}{x}\right)\right), \\
J_{1}(x) \sim \sqrt{\frac{2}{\pi x}}\left(-\sin \left(x-\frac{\pi}{4}\right)+O\left(\frac{1}{x}\right)\right),
\end{gathered}
$$

$$
Y_{1}(x) \sim \sqrt{\frac{2}{\pi x}}\left(\cos \left(x-\frac{\pi}{4}\right)+O\left(\frac{1}{x}\right)\right),
$$

so that the first order terms of the functions $\Psi_{e F}$ are

$$
\begin{gathered}
\Psi_{e I} \sim \frac{1}{2 \sqrt{\pi}}|H|^{-1 / 4} e^{\lambda S_{e}}, \\
\Psi_{e K} \sim \frac{1}{\sqrt{\pi}}|H|^{-1 / 4} e^{-\lambda S_{e}}, \\
\Psi_{e J} \sim-\frac{1}{\sqrt{\pi}}|H|^{-1 / 4} \sin \left(\lambda S_{e}-\frac{\pi}{4}\right), \\
\Psi_{e Y} \sim \frac{1}{\sqrt{\pi}}|H|^{-1 / 4} \cos \left(\lambda S_{e}-\frac{\pi}{4}\right) .
\end{gathered}
$$

We are now able to see how solutions of (50) evolve from $x>x_{e}$ to $x<x_{e}$. Given the connection rules of (78) and (79), we find first

$$
|H|^{-1 / 4} e^{\lambda S_{e}} \rightarrow 2|H|^{-1 / 4} \sin \left(\lambda\left|S_{e}\right|+\frac{\pi}{4}\right) .
$$

This is an analogous rule to the one of the turning points, but with the crucial difference that it is now the exponentially increasing solution the one connected to the sinus. This solution has no physical relevance, so we must choose the exponentially decreasing one to the right of $x_{e}$. This crosses to the left as

$$
\begin{aligned}
& |H|^{-1 / 4} e^{-\lambda S_{e}} \rightarrow-\frac{\pi}{2}\left(\cos \left(\lambda\left|S_{e}\right|+\frac{\pi}{4}\right)-i \sin \left(\lambda\left|S_{e}\right|+\frac{\pi}{4}\right)\right) \\
& \quad \text { if } m \phi_{e}^{\prime \prime}\left(x_{e}\right)<0,
\end{aligned}
$$

and

$$
\begin{aligned}
& |H|^{-1 / 4} e^{-\lambda S_{e}} \rightarrow-\frac{\pi}{2}\left(\cos \left(\lambda\left|S_{e}\right|+\frac{\pi}{4}\right)+i \sin \left(\lambda\left|S_{e}\right|+\frac{\pi}{4}\right)\right) \\
& \quad \text { if } m \phi_{e}^{\prime \prime}\left(x_{e}\right)>0 .
\end{aligned}
$$

As stated, in contrast to the turning point $x_{p}$, an imaginary part always occurs to the left of $x_{e}$ if the solution is decreasing as $x \rightarrow \infty$. In the alternative case $0<x_{e}<x_{p}<\infty$, the solution is oscillatory but real between $x_{e}$ and $x_{p}$, and an imaginary part develops to the left of $x_{e}$. For a more complex distribution of zeroes of $\phi_{e}^{\prime}-\omega / m$ and $\phi_{p}^{\prime}-\omega / m$, further connections are possible; always the solution is oscillatory whenever the two functions have different signs, nonoscillatory otherwise. The condition $\Psi_{e}(\infty)=0$ yields a onedimensional space of solutions. Thus, we may impose $\Psi_{e}(0)$ or $\Psi_{e}^{\prime}(0)$, but not both.

The case where the equilibrium velocities are the same is best considered independently. In this instance, Eq. (50) simplifies to

$$
\Psi_{e}^{\prime \prime}=\left(\lambda^{2}+g\right) \Psi_{e},
$$

where 


$$
g=m^{2}+\frac{\phi_{e}^{\prime \prime \prime}}{\phi_{e}^{\prime}-\omega / m} .
$$

For $H \neq 1$, the singular point of $g$ could be ignored because it coincides with the one of $H$, which is multiplied by the large constant $\lambda^{2}$. Since now the constant $\lambda^{2}$ has no relation to the singular point, there is no reason to expect rapid variation of the solutions and (97) may be treated by the Frobenius method. The indicial equation is $r(r-1)=0$, so one of the solutions has the form

$$
\Psi_{e 1}(x)=\sum_{k=1}^{\infty} c_{k}\left(x-x_{e}\right)^{k}
$$

and the other one

$$
\Psi_{e 2}(x)=f(x)+\Psi_{e 1}(x) \log \left(x-x_{e}\right), \quad f\left(x_{e}\right) \neq 0 .
$$

Outside a neighborhood of $x_{e}$ whose radius has order $1 / \lambda$, the solutions are to the first order a linear combination of $\exp (\lambda x)$ and $\exp (-\lambda x)$. The solution decreasing as $x \rightarrow \infty$ behaves like $\exp (-\lambda x)$ for $\lambda x$ large, becomes a linear combination of $\Psi_{e 1}$ and $\Psi_{e 2}$ at $\lambda\left|x-x_{e}\right|<\epsilon$, and extends to the left of $x_{e}$ as a linear combination of $\exp (\lambda x)$ and $\exp (-\lambda x)$. The important part is that the logarithmic term in (98) will again yield an imaginary part for $x<x_{e}$ : its sign depends as before on $\phi_{e}^{\prime \prime}\left(x_{e}\right)$. The relevance of this component of the solution concerning the absorption of energy at differentes parts of the plasma sheet will become evident in Sec. IV.

\section{BALANCE OF ENERGY}

We will show that a study of the mean absorption of electron kinetic energy will yield an invariant quantity which combines transfers from kinetic to magnetic energy and viceversa. This invariant is trivially equal to zero in the case where the solutions of (50) are real, i.e., when there is no zero of $\phi_{e}^{\prime}-\omega / m$ in the interval $[0, \infty)$. We start from the momentum equation (1) for the electrons, and in view of the results of Sec. III we feel justified in eliminating the ion velocity from the definition of the magnetic field in (5). The linearized equation takes the form

$$
\begin{aligned}
& \rho_{e} \frac{\partial \mathbf{u}_{e}}{\partial t}+\rho_{e} \mathbf{v}_{e} \cdot \nabla \mathbf{u}_{e}+\rho_{e} \mathbf{u}_{e} \cdot \nabla \mathbf{v}_{e} \\
& \quad=-N\left(\mathbf{e}+\mathbf{v}_{e} \times \mathbf{b}+\mathbf{u}_{e} \times \mathbf{B}\right)-\nabla p_{e} .
\end{aligned}
$$

Multiplying it by $\mathbf{u}_{e}$, we obtain

$$
\begin{aligned}
& \frac{1}{2} \rho_{e} \frac{\partial u_{e}^{2}}{\partial t}+\rho_{e} \mathbf{u}_{e} \cdot \nabla \mathbf{v}_{e} \cdot \mathbf{u}_{e}+\frac{1}{2} \rho_{e} \mathbf{v}_{e} \cdot \nabla u_{e}^{2} \\
& \quad=-N \mathbf{e} \cdot \mathbf{u}_{e}-N\left(\mathbf{v}_{e} \times \mathbf{b}\right) \cdot \mathbf{u}_{e}-\nabla p_{e} \cdot \mathbf{u}_{e}
\end{aligned}
$$

Since we have worked with the Fourier transforms in $y$ and $t$ of the original quantities, we should write down (100) for the Fourier transforms of $\mathbf{u}_{e}, \mathbf{e}, \mathbf{b}$ and $p_{e}$. An alternative easier method is to consider Fourier modes separately, i.e., to take functions of the form $a(x) \exp (i(\omega t+m y))$. Since for $f$ real one has

$$
\hat{f}(\omega, x, m)=\overline{\hat{f}(-\omega, x,-m)}
$$

we will take variables of the form

$$
h(x) e^{i(\omega t+m y)}+\overline{h(x)} e^{-i(\omega t+m y)},
$$

for $\omega \neq 0$. We choose them because for both terms of the expression in (102) the ratio $\omega / m$ is the same, so when substituting in Eq. (50) they will have the same turning points and singularities. We will integrate (100) in slabs $c<x<d$, with $y \in(-\infty, \infty)$ fixed. Apparently, there is a problem with this integration, since functions of the form (102) are not integrable in the variable $y$. However, note that in (100) there are always products of two functions in $y$; the remaining one, where it appears, depends only on $x$ as it corresponds to an equilibrium quantity. The Parseval theorem guarantees that, provided $f(y)$ is square integrable when $y \in(-\infty, \infty)$, so is $\hat{f}(m)$ when $m \in(-\infty, \infty)$. Moreover

$\int_{c}^{d} d x \int_{-\infty}^{\infty} f(x, y) \overline{g(x, y)} d x d y=\int_{c}^{d} d x \int_{-\infty}^{\infty} \hat{f}(x, m) \overline{\hat{g}(x, m)} d x d m$.

What we are really considering is each of the integrands

$$
\int_{c}^{d} \hat{f}(x, m) \overline{\hat{g}(x, m)} d x
$$

adding only the one for $m$ with the one for $-m$. The real dynamic variables are assumed to decrease fast enough in $y$ for the integral in (103) to be finite. For functions of the type (102), we have

$$
\hat{f}(m)=h(x) e^{i \omega t}
$$

so if $g$ is also of the type (102), with $c(x)$ instead of $h(x)$,

$$
\begin{aligned}
\hat{f}(x, m) \overline{\hat{g}(x, m)}+\hat{f}(x,-m) \overline{\hat{g}(x,-m)} & =h(x) \overline{c(x)}+\overline{h(x)} c(x) \\
& =2 \operatorname{Re}(h(x) \overline{c(x)}) .
\end{aligned}
$$

Even without taking Fourier transforms, we may cancel the integral of the third term

$$
\int_{c}^{d} \int_{-\infty}^{\infty} \frac{1}{2} \rho_{e} \mathbf{v}_{e} \cdot \nabla u_{e}^{2} d x d y=\int_{c}^{d} \int_{-\infty}^{\infty} \nabla \cdot\left(\frac{1}{2} \rho_{e} u_{e}^{2} \mathbf{v}_{e}\right) d x d y=0,
$$

by applying Gauss' theorem, since $\mathbf{v}_{e}$ has the direction of $\hat{y}$ and therefore it is orthogonal to the vector normal to the slabs, $\pm \hat{x}$.

All the functions in (100) will be written in terms of the expressions for the streamfunction $\psi_{e}$ and the pressure $p_{e}$. Let us denote

$$
\begin{aligned}
& \psi_{e}=a(x) e^{i(\omega t+m y)}+\overline{a(x)} e^{-i(\omega t+m y)}, \\
& p_{e}=p(x) e^{i(\omega t+m y)}+\overline{p(x)} e^{-i(\omega t+m y)} .
\end{aligned}
$$


$\mathbf{b}=b \hat{z}$ may be found through (26), which in our case reduces to

$$
\mathbf{b}=-N \psi e^{\hat{z}}=-N\left(a(x) e^{i(\omega t+m y)}+\overline{a(x)} e^{-i(\omega t+m y)}\right) \hat{z} .
$$

Hence

$$
\frac{\partial \mathbf{b}}{\partial t}=-N\left(i \omega a(x) e^{i(\omega t+m y)}-i \omega \overline{a(x)} e^{-i(\omega t+m y)}\right) \hat{z} .
$$

As for $\mathbf{e}=e \hat{y},(20)$ yields

$$
\frac{\partial b}{\partial t}=-\frac{\partial e}{\partial x} .
$$

Since we always assume that all the variables vanish at infinity, $e(x=\infty)=0$, we obtain from (111)

$$
e=N\left(i \omega A(x) e^{i(\omega t+m y)}-i \omega \overline{A(x)} e^{-i(\omega t+m y)}\right),
$$

where $A^{\prime}(x)=a(x), A(\infty)=0$, i.e.,

$$
A(x)=-\int_{x}^{\infty} a(s) d s .
$$

The integral in (114) will converge because $a$ decreases exponentially for large $x$. Finally, from (23)

$$
\begin{aligned}
\mathbf{u}_{e}= & \left(i m a(x) e^{i(\omega t+m y)}-i m \overline{a(x)} e^{-i(\omega t+m y)},\right. \\
& \left.-a^{\prime}(x) e^{i(\omega t+m y)}-\overline{a^{\prime}(x)} e^{-i(\omega t+m y)}, 0\right) .
\end{aligned}
$$

We may now integrate each term of (100) in the slab. First we notice

$$
\begin{gathered}
\hat{\mathbf{u}}_{e}(m)=\left(i m a, a^{\prime}, 0\right) e^{i \omega t}, \\
\frac{\partial \hat{\mathbf{u}}_{e}}{\partial t}(m)=\left(-\omega m a, i \omega a^{\prime}, 0\right) e^{i \omega t} .
\end{gathered}
$$

Therefore

$$
\rho_{e} \hat{\mathbf{u}}_{e}(m) \cdot \overline{\frac{\partial \hat{\mathbf{u}}_{e}}{\partial t}(m)}=i \rho_{e}\left(m^{2} \omega|a|^{2}-\omega\left|a^{\prime}\right|^{2}\right),
$$

whose real part is zero. According to (106), the integral is zero.

Let us consider now $N\left(\mathbf{v}_{e} \times \mathbf{b}\right) \cdot \mathbf{u}_{e}$. Since

$$
\begin{gathered}
\mathbf{v}_{e} \times \mathbf{b}=N\left(\phi_{e}^{\prime} \psi_{e}, 0,0\right), \\
\left(\mathbf{v}_{e} \times \mathbf{b}\right) \cdot \mathbf{u}_{e}=-N \phi_{e}^{\prime} \psi_{e} \frac{\partial \psi_{e}}{\partial y} .
\end{gathered}
$$

Now

$$
-N \phi_{e}^{\prime} \hat{\psi}_{e} \frac{\overline{\partial \psi_{e}}}{\partial y}=-i m \phi_{e}^{\prime}|a|^{2}
$$

whose real part is again zero. Hence we are left with

$$
\int_{c}^{d} \rho_{e} \mathbf{u}_{e} \cdot \nabla \mathbf{v}_{e} \cdot \mathbf{u}_{e}+N \mathbf{e} \cdot \mathbf{u}_{e}+\nabla \cdot\left(p_{e} \mathbf{u}_{e}\right) d x=0 .
$$

$$
\rho_{e} \mathbf{u}_{e} \cdot \nabla \mathbf{v}_{e}=-\left(0, \rho_{e} \phi_{e}^{\prime \prime}\left(i m a e^{i(\omega t+m y)}-i m \bar{a} e^{-i(\omega t+m y)}\right), 0\right),
$$

so that

$$
\left(\rho_{e} \hat{\mathbf{u}}_{e} \cdot \nabla \mathbf{v}_{e}\right)(m)=-\left(0, \rho_{e} \phi_{e}^{\prime \prime} i m a e^{i \omega t}, 0\right),
$$

and

$$
\left(\rho_{e} \hat{\mathbf{u}}_{e} \cdot \nabla \mathbf{v}_{e}\right)(m) \cdot \overline{\hat{\mathbf{u}}_{e}(m)}=\rho_{e} \phi_{e}^{\prime \prime} i m a \overline{a^{\prime}},
$$

so that when adding the term in $-m$ we are left with

$$
2 \rho_{e} \phi_{e}^{\prime \prime} \operatorname{Re}\left(i m a \overline{a^{\prime}}\right)=2 \rho_{e} \phi_{e}^{\prime \prime} m \operatorname{Im}\left(\bar{a} a^{\prime}\right) .
$$

Let us denote by $U$ and $V$ the real and imaginary parts of $a, a=U+i V$. Then

$$
\begin{aligned}
2 \rho_{e} \phi_{e}^{\prime \prime} m \operatorname{Im}\left(\bar{a} a^{\prime}\right) & =2 \rho_{e} \phi_{e}^{\prime \prime} m\left(U V^{\prime}-U^{\prime} V\right) \\
& =2 \rho_{e} \phi_{e}^{\prime \prime} m W(U, V),
\end{aligned}
$$

where $W(U, V)$ denotes the wronskian determinant. Since $U$ and $V$ are solutions of (50), which is a linear second-order equation without term in $\Psi_{e}^{\prime}$, the wronskian is constant at each side of the singularity $x_{e}$. For $x>x_{e}$, it is zero because there is not imaginary part there: $V=0$. For $x<x_{e}$, however, its value $W_{0}$ is not zero because of the generation of an imaginary part to the left of $x_{e}$. Thus, the integral

$$
I(c, d)=\int_{c}^{d} \rho_{e} \mathbf{u}_{e} \cdot \nabla \mathbf{v}_{e} \cdot \mathbf{u}_{e} d x
$$

equals

$$
\begin{gathered}
I(c, d)=2 \rho_{e} m W_{0}\left(\phi_{e}^{\prime}(d)-\phi_{e}^{\prime}(c)\right) \quad \text { for } d \leq x_{e}, \\
I(c, d)=2 \rho_{e} m W_{0}\left(\phi_{e}^{\prime}\left(x_{e}\right)-\phi_{e}^{\prime}(c)\right) \\
=2 \rho_{e} m W_{0}\left(\frac{\omega}{m}-\phi_{e}^{\prime}(c)\right) \quad \text { for } c \leq x_{e}<d, \\
I(c, d)=0 \text { for } c>x_{e} .
\end{gathered}
$$

Let us turn now to the integral of $N \mathbf{e} \cdot \mathbf{u}_{e}$ in 122 . Using 113 and (116), we find

$$
\begin{aligned}
N \hat{\mathbf{e}}(m) \cdot \overline{\mathbf{u}_{e}(m)} & =N^{2}\left(0, i \omega A e^{i \omega t}, 0\right) \cdot e^{-i \omega t}\left(-i m \bar{a}, \overline{a^{\prime}}, 0\right) \\
& =N^{2} i \omega A \overline{a^{\prime}} .
\end{aligned}
$$

Adding the term $-m$, the integrand becomes

$$
2 \operatorname{Re}\left(i \omega A \overline{a^{\prime}}\right)=2 \omega \operatorname{Im}\left(\bar{A} a^{\prime}\right) .
$$

Taking as before $a=U+i V$, let $R^{\prime}=U, S^{\prime}=V$, with $R(\infty)=S(\infty)=0$. Then the integrand may be written as

$$
\begin{aligned}
2 \omega N^{2}\left(R V^{\prime}-S U^{\prime}\right) & =2 \omega N^{2}\left(R S^{\prime \prime}-S R^{\prime \prime}\right) \\
& =2 \omega N^{2} \frac{d}{d x}\left(R S^{\prime}-S R^{\prime}\right) .
\end{aligned}
$$

Hence 


$$
\int_{c}^{d} N \mathbf{e} \cdot \mathbf{u}_{e} d x=2 \omega[W(R, S)(d)-W(R, S)(c)]
$$

where $W(R, S)$ denotes the wronskian of $R, S$

$$
W(R, S)(x)=U(x) \int_{x}^{\infty} V(s) d s-V(x) \int_{x}^{\infty} U(s) d s .
$$

In contrast with $W(U, V), W(R, S)$ is continuous in the whole interval $[0, \infty)$, and there is no need to make different cases as in (129)-(131). It is also zero for $x>x_{e}$. A first order approximation for $W(R, S)$ could be found by integration by parts of the WKB expression of $U$ and $V$, but only in the intervals where $|H|>r>0$, so it useless near the turning point and singularity.

Finally, by (109) and (116)

$$
\begin{gathered}
\hat{p}_{e}(m)=p e^{i \omega t} \\
\hat{\mathbf{u}}_{e}(m) \cdot \mathbf{n}= \pm i m a e^{i \omega t},
\end{gathered}
$$

where the \pm sign is positive in $x=d$ and negative in $x=c$. Thus the integral of the last term in (122) is

$$
\left.2 \operatorname{Re}(-i m p \bar{a})\right|_{c} ^{d}=\left.2 m \operatorname{Im}(p \bar{a})\right|_{c} ^{d},
$$

which again vanishes for $x>x_{e}$, since there both $p$ and $a$ are real. If we write $p=P+i Q$, this term equals

$$
2 m(Q U-P V)(d)-2 m(Q U-P V)(c) .
$$

Since (122) holds for any $c$ and $d$, and we have set this integral as a difference of terms in $d$ and $c$, we must conclude that the values of this term

$$
m \rho_{e} W(U, V) \phi_{e}^{\prime}+\omega N^{2} W(R, S)+m(Q U-P V),
$$

are the same for all points; thus it is constant for $x \in[0, \infty)$. Since the first term of (141) is discontinuous at $x_{e}$ because $W(U, V)$ goes from $W_{0}$ to zero, and the second one is continuous, the third one must be discontinuous at the singularity. Since $V$ is zero there, it must be $Q$, i.e., the imaginary part of the Fourier transform of the pressure which is discontinuous. Since all the terms in (141) are known except for the pressure, this identity may be viewed as defining it, or rather its phase, since we have the two components $P$ and $Q$ and a single equation. For $x>x_{e}$, (141) does not provide any information as all the terms are zero. The meaningful part of (141) is

$$
\rho_{e} W_{0}+\frac{\omega}{m} N^{2} W(R, S)+Q U-P V=\text { const, }
$$

which implies

$$
\frac{\omega}{m} N^{2} W(R, S)+Q U-P V=\text { const },
$$

for $x<x_{e}$.

\section{CONCLUSIONS}

To obtain dynamic equilibria in a multi-species collisionless plasma is an easy task when the quantities depend on a single spatial variable $x$ to which velocity and magnetic field are transversal, as well as among themselves. This corresponds to the cartesian equivalent of the geometry where the particles describe plane circles under a vertical magnetic field and a collinear electric one. Linear perturbations of these equilibria have a rich and intrincate structure where resonances with the equilibrium motion of the different species provide a plethora of different flow possibilities. We study the case where the plasma is formed by monoatomic ions, electrons, and neutral particles (which decouple from the rest and play no role in the analysis). Once a temporal frequency $\omega$ and a spatial one $m$ in the transversal variable are fixed, the Fourier transforms of the ion and electron streamfunctions satisfy a linear system with singularities whenever one of the equilibrium velocities equals $\omega / m$. By taking the inverse of the electron mass as a large parameter, one is able to use a WKB asymptotic method to show that the electron streamfunction dominates the ion one that the zeroes of the ion equilibrium velocity are turning points and those of the electron equilibrium velocity are singularities of the equation. The first order approximation to the solution possesses an exponential character at the intervals where the wave velocity $\omega / m$ is either larger or smaller than both equilibrium velocities, whereas it is oscillatory when it is larger than one and smaller than the other. Even if the solution is always real and exponentially decreasing for large values of $x$ (provided one assumes, plausibly enough, that the variables vanish at infinity), the solution always develops an imaginary part to the left of the singularities, which is essential for the balance of the different contributions to the electron kinetic energy. This balance yields an invariant of the system which provides information on the otherwise unknown electron pressure.

${ }^{1}$ D. Biskamp, E. Schwarz, and J. F. Drake, "Ion-controlled collisionless magnetic reconnection,” Phys. Rev. Lett. 75(21), 3850-3853 (1995).

${ }^{2}$ D. Biskamp, Magnetic Reconnection in Plasmas (Cambridge University Press, Cambridge, 2000).

${ }^{3}$ M. A. Shay, J. F. Drake, B. N. Rogers, and R. E. Denton, “Alfvénic collisionless magnetic reconnection and the Hall term,” J. Geophys. Res. 106, 3759-3772, doi:10.1029/1999JA001007 (2001).

${ }^{4}$ B. N. Rogers, R. E. Denton, J. F. Drake, and M. A. Shay, "Role of dispersive waves in collisionless magnetic reconnection," Phys. Rev. Lett. 87(19), 195004 (2001).

${ }^{5}$ B. T. Draine, W. G. Roberge, and A. Dalgarno, "Magnetohydrodynamic shock waves in molecular clouds,” Astrophys. J. 264, 485-507 (1983).

${ }^{6}$ B. P. Pandey and M. Wardle, "Hall magnetohydrodynamics of partially ionized plasmas," Month. Not. Roy. Astr. Soc. 385, 2269-2278 (2008).

${ }^{7}$ N. A. Krall and A. W. Trivelpiece, Principles of Plasma Physics (McGraw Hill, New York, 1973).

${ }^{8}$ F. F. Chen, Introduction to Plasma Physics and Controlled Fusion (Plenum Press, New York, 1983).

${ }^{9}$ S. I. Braginsky, “Transport processes in a plasma," Rev. Plasma Phys. 1, 205-311 (1965).

${ }^{10}$ L. Spitzer, Jr., Physics of Fully Ionized Plasmas (Interscience, New York, 1956).

${ }^{11}$ R. O. Dendy, Plasma Dynamics (Clarendon Press, Oxford, 1990).

${ }^{12}$ D. B. Melrose and R. C. McPhedran, Electromagnetic Processes in Dispersive Media (Cambridge University Press, Cambridge, 1991).

${ }^{13} \mathrm{R}$. Temam, Infinite-Dimensional Dynamical Systems in Mechanics and Physics (Springer, New York, 1988). 
${ }^{14}$ M. Núñez, "On the propagation of vorticity in multi-species plasmas," Phys. Plasmas 20, 122108 (2013).

${ }^{15}$ W. Wasow, Linear Turning Point Theory (Springer, New York, 1985).

${ }^{16} \mathrm{M}$. Bender and S. A. Orszag, Advanced Mathematical Methods for Scientists and Engineers I: Asymptotic Methods and Perturbation Theory, Springer (Berlin, 1999).
${ }^{17}$ M. V. Fedoryuk, Asymptotic Analysis: Linear Ordinary Differential Equations (Springer, Berlin, 1993).

${ }^{18}$ F. W. J. Olver, Asymptotics and Special Functions (AK Peters Ltd, Wellesley, Mass., 1997).

${ }^{19}$ Handbook of Mathematical Functions, edited by M. Abramowitz and I. A. Stegun (Dover, New York, 1970). 